\title{
Research and Application of a New Hybrid Forecasting Model Based on Genetic Algorithm Optimization: A Case Study of Shandong Wind Farm in China
}

\author{
Ping Jiang, ${ }^{1}$ Xiaofei Li, ${ }^{1}$ and Yao Dong ${ }^{2}$ \\ ${ }^{1}$ School of Statistics, Dongbei University of Finance and Economics, Dalian 116025, China \\ ${ }^{2}$ Department of Statistics, Florida State University, Tallahassee, FL 32306-4330, USA \\ Correspondence should be addressed to Xiaofei Li; lixiaofeiedu@gmail.com
}

Received 16 October 2014; Revised 19 December 2014; Accepted 20 December 2014

Academic Editor: Reza Jazar

Copyright (C) 2015 Ping Jiang et al. This is an open access article distributed under the Creative Commons Attribution License, which permits unrestricted use, distribution, and reproduction in any medium, provided the original work is properly cited.

With the increasing depletion of fossil fuel and serious destruction of environment, wind power, as a kind of clean and renewable resource, is more and more connected to the power system and plays a crucial role in power dispatch of hybrid system. Thus, it is necessary to forecast wind speed accurately for the operation of wind farm in hybrid system. In this paper, we propose a hybrid model called EEMD-GA-FAC/SAC to forecast wind speed. First, the Ensemble empirical mode decomposition (EEMD) can be applied to eliminate the noise of the original data. After data preprocessing, first-order adaptive coefficient forecasting method (FAC) or second-order adaptive coefficient forecasting method (SAC) can be employed to do forecast. It is significant to select optimal parameters for an effective model. Thus, genetic algorithm (GA) is used to determine parameter of the hybrid model. In order to verify the validity of the proposed model, every ten-minute wind speed data from three observation sites in Shandong Peninsula of China and several error evaluation criteria can be collected. Through comparing with traditional BP, ARIMA, FAC, and SAC model, the experimental results show that the proposed hybrid model EEMD-GA-FAC/SAC has the best forecasting performance.

\section{Introduction}

Wind, as a kind of environmentally friendly, economically competitive, and socially beneficial energy, has become the most widely used renewable energy resource all over the world. Particularly in China, the majority of energy sources are fossil fuels such as coal, oil, and natural gas, but rapid economic growth and decrease of fossil fuel reserves compel China to find out alternatives. Wind energy with more advantages including low cost of power generation, high degree of industrial maturity, and good physical and social environmental impact becomes the first choice of renewable energy sources in China [1]. Owing to the volatility and chaotic characteristics of wind speed, grid interconnection of wind farms has been a difficult and challenging task. However, in order to ensure the safe operation of the grid, the accuracy of wind speed forecasts plays a vital role in calculating the spinning reserve capacity of grid security forewarning management in wind grid. Therefore, it is necessary to forecast wind speed accurately. Generally speaking, there are two kinds of wind speed forecasts in terms of time span. One is long-term wind speed forecast, which is crucial for the sitting and sizing of wind power application $[2,3]$. It is helpful for wind risk evaluation. The other is shortterm wind speed forecast, which is significant to improve the efficiency of wind power generation systems $[4,5]$. The time scale of short-term forecasting range is from some seconds to minutes, hours, or several days. It can help the daily and intraday spot market, system management, and maintenance scheduling [6].

Many researchers have made efforts to develop good wind speed forecasting approaches including statistical methods, physical methods, physical-statistical models, artificial intelligent methods, and some other new hybrid methods. 
Statistical methods include autoregressive integrated moving average (ARIMA) model and generalized autoregressive conditional heteroskedasticity (GARCH) model. Kavasseri and Seetharaman [7] used f-ARIMA model to forecast wind speed of four sites in North Dakota on the day-ahead and two-dayahead horizons. Liu et al. [8] found that ARMA-GARCH ($\mathrm{M})$ models could improve the modelling sufficiency of mean wind speed as the height increases. Physical methods, like weather research forecasting model (WRF) and mesoscale models, combine multiple physical considerations and provide good forecasting accuracy. WRF model was evaluated by González-Mingueza and Muñoz-Gutiérrez [9] through different parameterization options in Peru to minimize the uncertainty of wind speed forecast. Janjai et al. [10] evaluated wind energy potential of Thailand using an atmospheric mesoscale model and a geographic information system (GIS) approach. The experimental performance presented areas in the south had high wind energy potential. For physicalstatistical methods, WRF results can usually be considered as input variables, combined with observed historical data, to train the system based on statistical theories [11]. Recently, some hybrid approaches based on artificial intelligence techniques have been proposed to forecast wind speed and have got good forecasting effects. Guo et al. [12] developed a hybrid model for wind speed prediction based on the empirical model decomposition (EMD) and feedforward neural network (FNN). Wang et al. [13] applied a combined epsilon-SVR forecast model based on the history data series eliminating the seasonal variation to make shortterm prediction. Pourmousavi Kani and Ardehali [14] applied artificial neural network (ANN) and Markov chain (MC) to propose a new hybrid ANN-MC model for short-term wind speed forecast. Guo et al. [15] proposed a hybrid seasonal autoregression integrated moving average and least square support vector machine (SARIMA-LSSVM) model to predict the mean monthly wind speed in Hexi Corridor. Wang et al. [16] used another new combined forecasting method including the seasonal ARIMA forecasting model, the seasonal exponential smoothing model, and the weighted support vector machines to make short-term prediction.

Empirical mode decomposition (EMD), widely adopted in many different fields [17-19], is a data adoptive method for analyzing nonlinear and nonstationary data. However, EMD may not function properly if the data does not meet certain conditions. Therefore, noise is introduced during the decomposition process to prevent mode mixing from contaminating the information embedded in IMFs [20]. This noise-assisted EMD method is named ensemble empirical mode decomposition (EEMD) [18]. Wind is an intermittent energy which means that there exists large variability due to temperature, humidity, pressure, and weather conditions [21]. Based on the above features, this work first employs EEMD method to eliminate high frequency fluctuant parts of tenminute wind speed. For the sake of improving the forecasting precision, optimized hybrid models need to be developed. A hybrid model including first-order adaptive coefficient forecasting method (FAC) and second-order adaptive coefficient forecasting method (SAC) is proposed in this paper. In addition, the genetic algorithm (GA) is a highly parallel, stochastic, and adaptive optimization technique on the basis of biological genetic evolutionary mechanisms. Its performing operation is similar to natural selection, crossover, and mutation to get the final optimization results after repeated iterations [22].

The structure of this paper is as follows. Section 2 refers to our contribution of the paper. Section 3 introduces relative methods including ensemble empirical mode decomposition (EEMD), genetic algorithm (GA), first-order adaptive coefficient forecasting method (FAC), and second-order adaptive coefficient forecasting method (SAC). In Section 4, experimental simulation and evaluation of forecasting performance can be described in detail. Finally, Section 5 concludes this work.

\section{Our Contributions}

We propose an intelligent optimized hybrid model EEMDGA-FAC/SAC based on the EEMD, GA, and FAC/SAC model which have several advantages. To begin with, as an intermittent energy, wind is vulnerable to the impact of temperature, humidity, pressure, and weather conditions, causing its characteristic of nonstationary and high frequency. It is necessary to develop methods of eliminating the interference information that would be well practical in application. Second, through the performance of experimental simulation results, it is obviously illustrated that the proposed hybrid model EEMD-GA-FAC/SAC is suitable for the current research. Second, ten-minute wind speed data is nonlinear and nonstationary. The EEMD-GA-FAC/SAC model can effectively eliminate high frequency inference signals and determine the optimal weight parameters of FAC and SAC model. Third, in order to select the most suitable weight coefficients for the hybrid model, GA is applied to determine the weight parameters of FAC or SAC. Finally, from the case study it can be concluded that the MAPE, MRE, and MAE of the proposed EEMD-GA-FAC/SAC model are smaller than the ones of the EEMD-FAC/SAC, FAC/SAC, BP, and ARIMA model. To sum up, the hybrid model EEMD-GA-FAC/SAC has good forecasting quality and high forecasting accuracy. For the above reasons, the proposed hybrid model EEMDGA-FAC/SAC is more effective and adaptive to improve the forecasting accuracy than traditional BP, ARIMA, FAC, and SAC model.

\section{Relative Methods}

3.1. Ensemble Empirical Mode Decomposition (EEMD). Empirical mode decomposition (EMD) is an adaptive and efficient approach that is used to decompose nonlinear and nonstationary signals into a series of meaningful IMFs and one residual trend from high frequency to low frequency $[23,24]$. However, the mode mixing problem is the most important shortcoming of EMD, which indicates either a single IMF consisting of signals of dramatically disparate scales or a signal of the same scale appearing in different IMF components, and usually intermittency of analyzing signal [25]. In order to eliminate the mode mixing phenomenon and get the actual time-frequency distribution of the seismic 
TABLE 1: The forecasting results of four traditional models for original data.

\begin{tabular}{|c|c|c|c|c|c|c|c|c|c|c|}
\hline \multirow[b]{2}{*}{ Data } & \multirow[b]{2}{*}{ Time } & \multirow{2}{*}{$\begin{array}{l}\text { Actual } \\
\text { value } \\
(\mathrm{m} / \mathrm{s})\end{array}$} & \multicolumn{2}{|c|}{$\operatorname{FAC}(\beta=0.2)$} & \multicolumn{2}{|c|}{$\operatorname{SAC}(\beta=0.2)$} & \multicolumn{2}{|c|}{$\mathrm{BP}$} & \multicolumn{2}{|c|}{ ARIMA } \\
\hline & & & $\begin{array}{l}\text { Forecasting } \\
\text { value }(\mathrm{m} / \mathrm{s})\end{array}$ & $\begin{array}{c}\text { MAPE } \\
(\%)\end{array}$ & $\begin{array}{l}\text { Forecasting } \\
\text { value }(\mathrm{m} / \mathrm{s})\end{array}$ & $\begin{array}{c}\text { MAPE } \\
(\%)\end{array}$ & $\begin{array}{l}\text { Forecasting } \\
\text { value }(\mathrm{m} / \mathrm{s})\end{array}$ & $\begin{array}{l}\text { MAPE } \\
(\%)\end{array}$ & $\begin{array}{l}\text { Forecasting } \\
\text { value }(\mathrm{m} / \mathrm{s})\end{array}$ & $\begin{array}{c}\text { MAPE } \\
(\%)\end{array}$ \\
\hline \multirow{12}{*}{$\begin{array}{l}\text { Observation } \\
\text { site } 1\end{array}$} & 0:00 & 6.7 & 6.63 & 1.03 & 6.69 & 0.15 & 7.24 & 8.03 & 7.18 & 7.18 \\
\hline & $2: 00$ & 8.4 & 7.76 & 7.58 & 7.74 & 7.81 & 8.00 & 4.80 & 7.96 & 5.29 \\
\hline & 4:00 & 8.5 & 7.86 & 7.48 & 7.84 & 7.77 & 8.00 & 5.92 & 8.00 & 5.91 \\
\hline & $6: 00$ & 6.9 & 7.14 & 3.52 & 7.15 & 3.55 & 7.08 & 2.68 & 7.22 & 4.57 \\
\hline & 8:00 & 6.8 & 6.67 & 1.92 & 6.73 & 1.02 & 6.16 & 9.34 & 6.28 & 7.70 \\
\hline & $10: 00$ & 6.9 & 6.86 & 0.53 & 6.80 & 1.51 & 7.16 & 3.73 & 7.15 & 3.58 \\
\hline & $12: 00$ & 8.3 & 7.75 & 6.66 & 7.73 & 6.84 & 7.50 & 9.61 & 7.42 & 10.59 \\
\hline & $14: 00$ & 10.6 & 10.67 & 0.63 & 10.67 & 0.65 & 10.47 & 1.19 & 10.26 & 3.23 \\
\hline & $16: 00$ & 9.6 & 9.79 & 1.96 & 9.81 & 2.18 & 10.42 & 8.56 & 9.89 & 2.98 \\
\hline & 18:00 & 11.7 & 11.81 & 0.94 & 10.67 & 8.77 & 11.66 & 0.36 & 11.96 & 2.20 \\
\hline & $20: 00$ & 10.3 & 10.52 & 2.10 & 10.52 & 2.16 & 10.41 & 1.04 & 10.06 & 2.38 \\
\hline & $22: 00$ & 12 & 9.41 & 21.58 & 9.41 & 21.57 & 9.90 & 17.52 & 9.82 & 18.19 \\
\hline \multirow{12}{*}{$\begin{array}{l}\text { Observation } \\
\text { site } 2\end{array}$} & 0:00 & 7.3 & 6.56 & 10.09 & 6.55 & 10.25 & 6.69 & 8.31 & 6.57 & 10.03 \\
\hline & $2: 00$ & 8.5 & 8.17 & 3.88 & 8.16 & 3.96 & 8.29 & 2.47 & 8.36 & 1.59 \\
\hline & 4:00 & 8.1 & 7.96 & 1.71 & 7.90 & 2.43 & 8.10 & 0.04 & 7.90 & 2.43 \\
\hline & $6: 00$ & 8 & 8.35 & 4.39 & 8.32 & 4.04 & 8.41 & 5.14 & 8.28 & 3.52 \\
\hline & 8:00 & 7.2 & 6.48 & 9.99 & 6.49 & 9.84 & 6.62 & 8.11 & 6.98 & 3.07 \\
\hline & $10: 00$ & 7 & 7.01 & 0.15 & 6.96 & 0.56 & 7.34 & 4.90 & 6.79 & 3.03 \\
\hline & $12: 00$ & 8.4 & 7.74 & 7.85 & 7.73 & 7.97 & 7.21 & 14.21 & 7.36 & 12.38 \\
\hline & $14: 00$ & 11.6 & 10.65 & 8.16 & 10.61 & 8.51 & 10.83 & 6.65 & 10.58 & 8.78 \\
\hline & $16: 00$ & 8.9 & 9.59 & 7.74 & 9.63 & 8.16 & 9.53 & 7.12 & 10.02 & 12.56 \\
\hline & 18:00 & 10.9 & 10.02 & 8.03 & 10.04 & 7.85 & 11.19 & 2.68 & 10.72 & 1.61 \\
\hline & $20: 00$ & 10.6 & 11.19 & 5.54 & 11.21 & 5.72 & 10.73 & 1.21 & 11.01 & 3.91 \\
\hline & $22: 00$ & 11.4 & 12.27 & 7.60 & 12.31 & 8.02 & 12.52 & 9.79 & 12.19 & 6.93 \\
\hline \multirow{12}{*}{$\begin{array}{l}\text { Observation } \\
\text { site } 3\end{array}$} & $0: 00$ & 8.3 & 9.34 & 12.49 & 9.37 & 12.83 & 8.91 & 7.32 & 8.89 & 7.05 \\
\hline & $2: 00$ & 9.4 & 8.86 & 5.69 & 8.84 & 5.92 & 9.20 & 2.13 & 8.99 & 4.35 \\
\hline & 4:00 & 9.2 & 9.24 & 0.45 & 9.21 & 0.09 & 9.46 & 2.85 & 9.36 & 1.79 \\
\hline & $6: 00$ & 8.1 & 8.15 & 0.59 & 8.15 & 0.65 & 8.29 & 2.29 & 8.15 & 0.56 \\
\hline & 8:00 & 8.3 & 8.47 & 2.04 & 8.47 & 2.00 & 8.69 & 4.74 & 8.50 & 2.40 \\
\hline & $10: 00$ & 7.1 & 7.82 & 10.08 & 7.79 & 9.65 & 7.84 & 10.38 & 7.88 & 10.93 \\
\hline & $12: 00$ & 8.8 & 8.68 & 1.35 & 8.68 & 1.40 & 9.01 & 2.33 & 8.71 & 0.98 \\
\hline & $14: 00$ & 10.3 & 9.90 & 3.91 & 9.94 & 3.52 & 9.86 & 4.28 & 9.38 & 8.95 \\
\hline & $16: 00$ & 10.1 & 11.04 & 9.31 & 11.01 & 9.04 & 11.97 & 18.55 & 11.57 & 14.57 \\
\hline & 18:00 & 12.2 & 11.23 & 7.94 & 11.22 & 8.05 & 11.60 & 4.91 & 11.42 & 6.37 \\
\hline & $20: 00$ & 12.2 & 11.62 & 4.79 & 11.61 & 4.87 & 11.68 & 4.29 & 11.82 & 3.14 \\
\hline & $22: 00$ & 11 & 10.74 & 2.32 & 10.70 & 2.72 & 11.48 & 4.33 & 11.26 & 2.37 \\
\hline
\end{tabular}

signal, a new approach called ensemble empirical mode decomposition (EEMD) was proposed $[26,27]$. The aim of EEMD is to add white noise to the data, which distributes uniformly in the whole time-frequency space, and make the bits of signals of different scales be automatically designed onto proper scales of reference established by the white noise [25]. The detailed description of the algorithm of EEMD is as follows [24]. 
TABLE 2: Errors comparisons of four traditional models.

\begin{tabular}{lcccc}
\hline & Models & MAPE $(\%)$ & MSE $\left(\mathrm{m}^{2} / \mathrm{s}^{2}\right)$ & $\mathrm{MAE}(\mathrm{m} / \mathrm{s})$ \\
\hline \multirow{4}{*}{ Observation } & BP & 6.0643 & 0.5808 & 0.5432 \\
site 1 & ARIMA & 6.1497 & 0.5814 & 0.5588 \\
& FAC & 4.6603 & 0.6668 & 0.4562 \\
& SAC & 5.3317 & 0.7595 & 0.5356 \\
\hline \multirow{4}{*}{ Observation } & BP & 5.8862 & 0.4006 & 0.5245 \\
site 2 & ARIMA & 5.8202 & 0.4135 & 0.5280 \\
& FAC & 6.2611 & 0.4150 & 0.5758 \\
Observation & ARIMA & 5.2878 & 0.4184 & 0.5066 \\
site 3 & BAC & 6.4436 & 0.4339 & 0.5927 \\
& FAC & 5.0800 & 0.3593 & 0.4847 \\
& SAC & 5.0617 & 0.3610 & 0.4840 \\
\hline
\end{tabular}

Step 1. Initialize the number of ensemble $M$ and the amplitude of the added white noise, with $m=1$.

Step 2. Perform the $m$ th trial on the signal added with white noise:

(a) add a white noise series with the given amplitude to the investigated signal

$$
x_{m}(t)=x(t)+n_{m}(t)
$$

where $n_{m}(t)$ represents the $m$ th added white noise series and $x_{m}(t)$ indicates the noise-added signal of the $m$ th trial;

(b) decompose the noise-added signal $x_{m}(t)$ into several IMFs $c_{i, m}(i=1,2, \ldots, I)$ by the EMD method, where $c_{i, m}$ denotes the $i$ th IMF of the $m$ th trial and $I$ is the number of IMFs;

(c) if $m<M$ then go to step (a) with $m=m+1$. Repeat steps (a) and (b) again and again, but with different white noise series each time.

Step 3. Calculate the ensemble mean $a_{i}$ of the $M$ trials for each IMF:

$$
a_{i}=\frac{1}{M} \sum_{m=1}^{M} c_{i, m} \quad i=1,2, \ldots, I ; m=1,2, \ldots, M .
$$

Step 4. Report the mean $a_{i}(i=1,2, \ldots, I)$ of each of the IMFs as the final IMFs.

3.2. Genetic Algorithm (GA). The genetic algorithm (GA), a famous metaheuristic algorithm, can follow the natural evolution processes. The GA starts at defining optimization variables, objective functions, and control parameters [28]. Generally speaking, the GA begins by creating a random population which composes of a certain number of individual solutions indicated by "chromosomes", and the "chromosomes" contain all the genes (i.e., variables) and are involved in each possible solution. The chromosomes are evaluated based on the "objective function," which is the expected objective of the problem [29]. The brief procedure can be seen in the following way $[30,31]$.

Step 1. Randomly generating the initial population.

Step 2. Computing and saving the fitness function for each individual in the population. The individual fitness function can be defined as $F(i)=\max f-f(i) ; f$ is the objective function.

Step 3. Section operation: the fitness value in the population can take part in this operation on the basis of probabilities. Define selection probabilities of each individual while maintaining the proportionality. In the selection operation, the members of the population with better fitness value can participate several times, while the members with worse value may be removed for the sake of getting a larger fitness average. Next, we can generate offspring.

Step 4. Crossover operation: it allows an exchange of the design characteristics between two mating parents. This operation is done by selecting two mating parents in which two random places are selected on each chromosome string and the strings between these two places among the mates are exchanged.

Step 5. Mutation operation: the aim is to search the minimum solution and keep population diversity and avoid the premature convergence phenomenon. It is invoked with a low probability at a randomly selected site on the chromosomal string of the randomly chosen design. The operation consists of a switching of a 0 to 1 or vice versa.

\subsection{First-Order Adaptive Coefficient Forecasting Method} (FAC). The aim of the first-order adaptive coefficient forecasting method (FAC) $[32,33]$ is to correct the coefficient values constantly on the basis of changes in data, thus making forecasting results the best. The forecasting equation is as follows:

$$
\begin{aligned}
\widehat{x}_{t+1} & =\alpha_{t} x_{t}+\left(1-\alpha_{t}\right) \widehat{x}_{t} \\
& =\widehat{x}_{t}+\alpha_{t}\left(x_{t}-\widehat{x}_{t}\right) \\
& =\widehat{x}_{t}+\alpha_{t} e_{t} .
\end{aligned}
$$

The solution of $\alpha_{t}$ is given below. If there is a system error during a particular forecasting time (it means all the $e_{t}$ values are positive or negative), $\alpha_{t}$ should be larger. When the forecasting value $\widehat{x}_{t}$ is relatively smaller, that is, $e_{t}=x_{t}-\widehat{x}_{t}>$ 0 , we can make $\alpha_{t}$ larger so as to increase $\hat{x}_{t+1}$ according to (3). When the forecasting value $\hat{x}_{t}$ is relatively larger, that is, $e_{t}=x_{t}-\widehat{x}_{t}<0$, we can also make $\alpha_{t}$ larger in order to decrease $\widehat{x}_{t+1}$. In other words, the larger the system error is, the larger the $\alpha_{t}$ value is. If there is not a system error, that is, values of $e_{t}$ are alternately positive and negative and absolute values of $e_{t}$ are relatively smaller, thus $\alpha_{t}$ can remain unchanged. How to measure the system error? We can give measure methods in the following way. 
TABLE 3: The forecasting results of EEMD-FAC/SAC model in three sites.

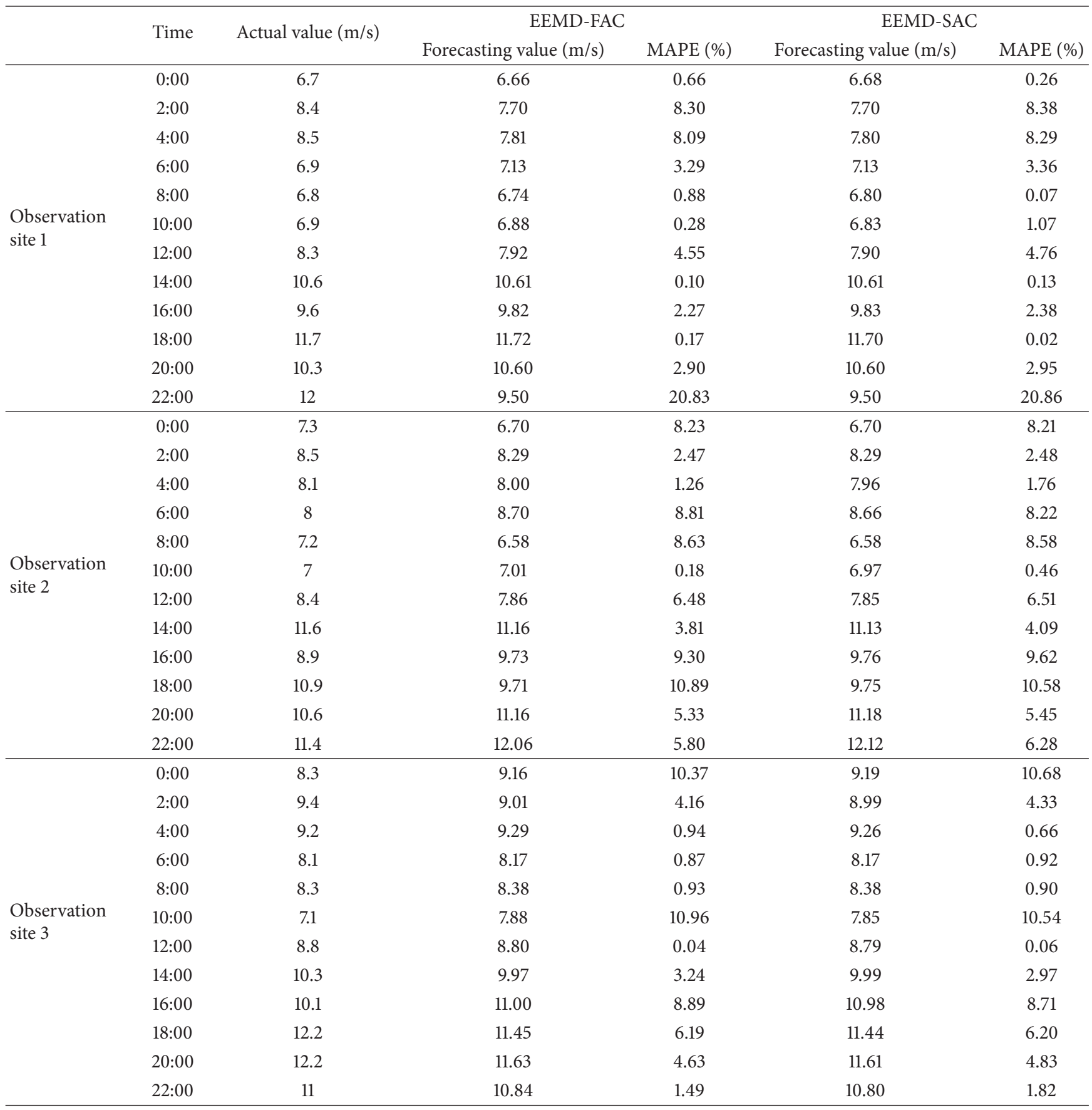

Supposing that $\beta$ is a constant $(0<\beta<1)$, we can make exponentially weighted average for the forecasting errors $e_{K}(K=1,2, \ldots, t)$ before time $t$ :

$$
E_{t}=\beta e_{t}+\beta(1-\beta) e_{t-1}+\cdots+\beta(1-\beta)^{t-1} e_{1} .
$$

$\left|E_{t}\right|$ can reflect the situation of the forecasting system errors; when $t=t-1$,

$$
E_{t-1}=\beta e_{t-1}+\beta(1-\beta) e_{t-2}+\cdots+\beta(1-\beta)^{t-2} e_{1} .
$$

Thus, based on (4) and (5) the recursive calculative formula of $E_{t}$ can be got as follows:

$$
\begin{aligned}
E_{t} & =\beta e_{t}+\beta(1-\beta) e_{t-1}+\cdots+\beta(1-\beta)^{t-1} e_{1} \\
& =\beta e_{t}+(1-\beta)\left[\beta e_{t-1}+\cdots+\beta(1-\beta)^{t-2} e_{1}\right] \\
& =\beta e_{t}+(1-\beta) E_{t-1} .
\end{aligned}
$$

In order to make $0<\alpha_{t}<1$, let

$$
M_{t}=\beta\left|e_{t}\right|+\beta(1-\beta)\left|e_{t-1}\right|+\cdots+\beta(1-\beta)^{t-1}\left|e_{1}\right| ;
$$


TABLE 4: The forecasting results and optimized parameters of the proposed hybrid model EEMD-GA-FAC/SAC in site 1.

\begin{tabular}{|c|c|c|c|c|c|c|c|c|}
\hline & & $\beta$ & Time & Actual value $(\mathrm{m} / \mathrm{s})$ & $\begin{array}{l}\text { Forecasting } \\
\text { value }(\mathrm{m} / \mathrm{s})\end{array}$ & MAPE (\%) & $\operatorname{MSE}\left(\mathrm{m}^{2} / \mathrm{s}^{2}\right)$ & $\operatorname{MAE}(\mathrm{m} / \mathrm{s})$ \\
\hline \multirow{24}{*}{$\begin{array}{l}\text { Observation } \\
\text { site } 1\end{array}$} & \multirow{10}{*}{ EEMD-GA-FAC } & 0.1729 & $0: 00$ & 6.7 & 6.70 & 0.00 & 0.0000 & 0.0001 \\
\hline & & 0.0000 & $2: 00$ & 8.4 & 7.77 & 7.50 & 0.3974 & 0.6304 \\
\hline & & 0.5587 & 4:00 & 8.5 & 7.93 & 6.75 & 0.3293 & 0.5739 \\
\hline & & 0.0000 & $6: 00$ & 6.9 & 7.10 & 2.89 & 0.0396 & 0.1991 \\
\hline & & 0.1777 & $8: 00$ & 6.8 & 6.80 & 0.00 & 0.0000 & 0.0000 \\
\hline & & 0.2059 & $10: 00$ & 6.9 & 6.90 & 0.00 & 0.0000 & 0.0000 \\
\hline & & 0.9994 & $12: 00$ & 8.3 & 8.01 & 3.47 & 0.0828 & 0.2877 \\
\hline & & 0.1445 & $14: 00$ & 10.6 & 10.60 & 0.00 & 0.0000 & 0.0000 \\
\hline & & 0.0000 & $16: 00$ & 9.6 & 9.76 & 1.66 & 0.0253 & 0.1591 \\
\hline & & 0.1927 & $18: 00$ & 11.7 & 11.70 & 0.00 & 0.0000 & 0.0001 \\
\hline & \multirow{14}{*}{ EEMD-GA-SAC } & 0.0000 & 20:00 & 10.3 & 10.33 & 0.34 & 0.0012 & 0.0345 \\
\hline & & 0.0191 & $22: 00$ & 12 & 10.22 & 14.80 & 3.1527 & 1.7756 \\
\hline & & 0.1889 & $0: 00$ & 6.7 & 6.70 & 0.00 & 0.0000 & 0.0001 \\
\hline & & 0.0000 & $2: 00$ & 8.4 & 8.03 & 4.39 & 0.1363 & 0.3692 \\
\hline & & 0.5637 & 4:00 & 8.5 & 7.93 & 6.75 & 0.3295 & 0.5740 \\
\hline & & 0.0000 & $6: 00$ & 6.9 & 7.01 & 1.61 & 0.0124 & 0.1113 \\
\hline & & 0.1981 & 8:00 & 6.8 & 6.80 & 0.00 & 0.0000 & 0.0000 \\
\hline & & 0.2228 & $10: 00$ & 6.9 & 6.90 & 0.00 & 0.0000 & 0.0001 \\
\hline & & 0.0000 & $12: 00$ & 8.3 & 8.02 & 3.40 & 0.0795 & 0.2819 \\
\hline & & 0.1386 & $14: 00$ & 10.6 & 10.60 & 0.00 & 0.0000 & 0.0000 \\
\hline & & 0.0018 & $16: 00$ & 9.6 & 9.58 & 0.23 & 0.0005 & 0.0217 \\
\hline & & 0.1993 & $18: 00$ & 11.7 & 11.70 & 0.00 & 0.0000 & 0.0000 \\
\hline & & 1.0000 & $20: 00$ & 10.3 & 10.33 & 0.34 & 0.0012 & 0.0345 \\
\hline & & 0.0201 & $22: 00$ & 12 & 10.25 & 14.61 & 3.0736 & 1.7532 \\
\hline
\end{tabular}

when $t=t-1$,

$$
M_{t-1}=\beta\left|e_{t-1}\right|+\beta(1-\beta)\left|e_{t-2}\right|+\cdots+\beta(1-\beta)^{t-2}\left|e_{1}\right|
$$

In a similar way, the recursive calculative formula of $M_{t}$ can be obtained:

$$
\begin{aligned}
M_{t} & =\beta\left|e_{t}\right|+\beta(1-\beta)\left|e_{t-1}\right|+\cdots+\beta(1-\beta)^{t-1}\left|e_{1}\right| \\
& =\beta\left|e_{t}\right|+(1-\beta)\left[\beta\left|e_{t-1}\right|+\cdots+\beta(1-\beta)^{t-2}\left|e_{1}\right|\right] \\
& =\beta\left|e_{t}\right|+(1-\beta) M_{t-1} .
\end{aligned}
$$

In order to satisfy the above requirements, let $\alpha_{t}=$ $\left|E_{t}\right| / M_{t}$. Figure 1 shows the calculation procedure of firstorder adaptive coefficient forecasting method.

\subsection{Second-Order Adaptive Coefficient Forecasting Method} $(S A C)$. The principle and calculation formula of secondorder adaptive coefficient forecasting method (SAC) is the same as the first-order adaptive coefficient method (FAC), so the calculation process (shown in Figure 1) can be given directly as follows $[32,34]$.

Step 1. Initializing parameters: let $\beta=0.1$ or $\beta=0.2, S_{0}^{(1)}=$ $S_{0}^{(2)}=x_{1}, M_{0}=0, E_{0}=0$, and $\widehat{x}_{1}=x_{1}$. So it can be easily calculated that $E_{1}=0$ and $M_{1}=0$ by (10), then at this time $\alpha_{1}=0$ which is put in (11), $S_{1}^{(1)}=S_{1}^{(2)}=x_{1}$.

Step 2. Calculating adaptive coefficient $\alpha_{t}$ according to

$$
\begin{gathered}
e_{t}=x_{t}-\widehat{x}_{t}, \\
E_{t}=\beta e_{t}+(1-\beta) E_{t-1}, \\
M_{t}=\beta\left|e_{t}\right|+(1-\beta) M_{t-1}, \\
\alpha_{t}=\frac{\left|E_{t}\right|}{M_{t}},
\end{gathered}
$$

where $e_{t}$ is the forecasting error, $E_{t}$ is the first-order exponential smoothing of the error series $e_{1}, e_{2}, \ldots, e_{t}$, and $M_{t}$ is the first-order exponential smoothing of the absolute error series $\left|e_{1}\right|,\left|e_{2}\right|, \ldots,\left|e_{t}\right|$.

Step 3. Make prediction on the basis of

$$
\begin{gathered}
S_{t}^{(1)}=\alpha_{t} x_{t}+\left(1-\alpha_{t}\right) S_{t-1}^{(1)}, \\
S_{t}^{(2)}=\alpha_{t} S_{t}^{(1)}+\left(1-\alpha_{t}\right) S_{t-1}^{(2)} \\
\widehat{a}_{t}=2 S_{t}^{(1)}-S_{t}^{(2)},
\end{gathered}
$$


TABLE 5: The forecasting results and optimized parameters of the proposed hybrid model EEMD-GA-FAC/SAC in site 2.

\begin{tabular}{|c|c|c|c|c|c|c|c|c|}
\hline & & $\beta$ & Time & Actual value $(\mathrm{m} / \mathrm{s})$ & $\begin{array}{l}\text { Forecasting } \\
\text { value }(\mathrm{m} / \mathrm{s})\end{array}$ & MAPE (\%) & $\operatorname{MSE}\left(\mathrm{m}^{2} / \mathrm{s}^{2}\right)$ & $\operatorname{MAE}(\mathrm{m} / \mathrm{s})$ \\
\hline \multirow{24}{*}{$\begin{array}{l}\text { Observation } \\
\text { site } 2\end{array}$} & \multirow{10}{*}{ EEMD-GA-FAC } & 0.0425 & $0: 00$ & 7.3 & 6.99 & 4.31 & 0.0992 & 0.3149 \\
\hline & & 0.9957 & $2: 00$ & 8.5 & 8.30 & 2.34 & 0.0395 & 0.1988 \\
\hline & & 0.2433 & $4: 00$ & 8.1 & 8.10 & 0.00 & 0.0000 & 0.0001 \\
\hline & & 0.0372 & $6: 00$ & 8 & 8.23 & 2.89 & 0.0536 & 0.2315 \\
\hline & & 0.0187 & $8: 00$ & 7.2 & 7.20 & 0.02 & 0.0000 & 0.0013 \\
\hline & & 0.1959 & $10: 00$ & 7 & 7.00 & 0.00 & 0.0000 & 0.0001 \\
\hline & & 0.2481 & $12: 00$ & 8.4 & 7.90 & 5.89 & 0.2450 & 0.4950 \\
\hline & & 0.0000 & $14: 00$ & 11.6 & 11.42 & 1.59 & 0.0342 & 0.1849 \\
\hline & & 0.0000 & $16: 00$ & 8.9 & 9.57 & 7.48 & 0.4428 & 0.6654 \\
\hline & & 1.0000 & 18:00 & 10.9 & 10.40 & 4.62 & 0.2541 & 0.5041 \\
\hline & \multirow{14}{*}{ EEMD-GA-SAC } & 0.8061 & $20: 00$ & 10.6 & 10.92 & 3.06 & 0.1052 & 0.3243 \\
\hline & & 0.0000 & $22: 00$ & 11.4 & 11.45 & 0.45 & 0.0026 & 0.0508 \\
\hline & & 0.6201 & $0: 00$ & 7.3 & 6.72 & 7.98 & 0.3394 & 0.5826 \\
\hline & & 1.0000 & $2: 00$ & 8.5 & 8.39 & 1.28 & 0.0118 & 0.1086 \\
\hline & & 0.2561 & 4:00 & 8.1 & 8.10 & 0.00 & 0.0000 & 0.0000 \\
\hline & & 0.0379 & $6: 00$ & 8 & 8.19 & 2.35 & 0.0354 & 0.1882 \\
\hline & & 0.3939 & 8:00 & 7.2 & 6.63 & 7.98 & 0.3301 & 0.5746 \\
\hline & & 0.0209 & $10: 00$ & 7 & 6.58 & 6.04 & 0.1786 & 0.4226 \\
\hline & & 0.0149 & $12: 00$ & 8.4 & 7.05 & 16.12 & 1.8333 & 1.3540 \\
\hline & & 0.0210 & $14: 00$ & 11.6 & 10.59 & 8.70 & 1.0177 & 1.0088 \\
\hline & & 0.9990 & $16: 00$ & 8.9 & 9.47 & 6.45 & 0.3300 & 0.5745 \\
\hline & & 1.0000 & $18: 00$ & 10.9 & 11.05 & 1.42 & 0.0240 & 0.1548 \\
\hline & & 1.0000 & $20: 00$ & 10.6 & 10.96 & 3.41 & 0.1306 & 0.3614 \\
\hline & & 0.0000 & 22:00 & 11.4 & 11.39 & 0.06 & 0.0001 & 0.0073 \\
\hline
\end{tabular}

FAC
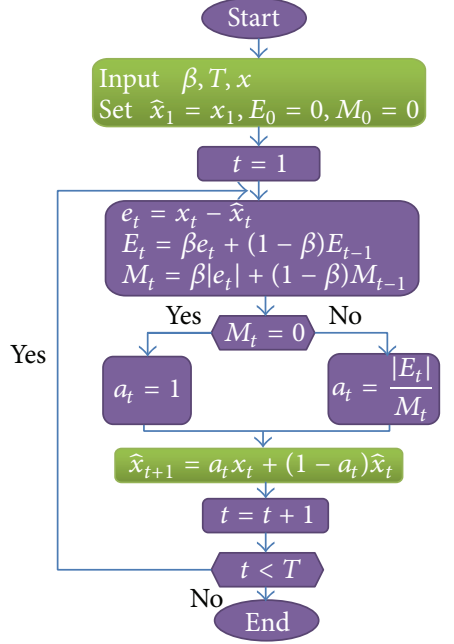

Special part of FAC

Common part

Special part of SAC
SAC
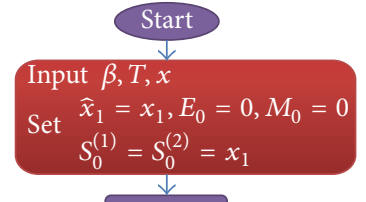

$t=1$
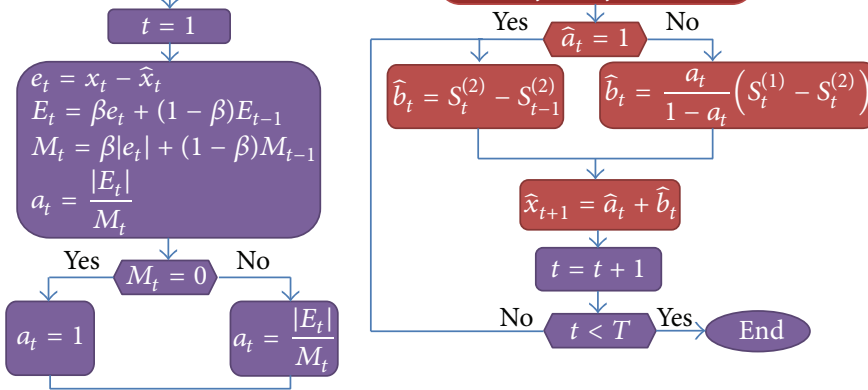

Figure 1: Flow charts of FAC and SAC. 
TABLE 6: The forecasting results and optimized parameters of the proposed hybrid model EEMD-GA-FAC/SAC in site 3.

\begin{tabular}{|c|c|c|c|c|c|c|c|c|}
\hline & & $\beta$ & Time & Actual value $(\mathrm{m} / \mathrm{s})$ & $\begin{array}{l}\text { Forecasting } \\
\text { value }(\mathrm{m} / \mathrm{s})\end{array}$ & MAPE (\%) & $\operatorname{MSE}\left(\mathrm{m}^{2} / \mathrm{s}^{2}\right)$ & $\operatorname{MAE}(\mathrm{m} / \mathrm{s})$ \\
\hline \multirow{24}{*}{$\begin{array}{l}\text { Observation } \\
\text { site } 3\end{array}$} & \multirow{10}{*}{ EEMD-GA-FAC } & 0.9996 & $0: 00$ & 8.3 & 8.87 & 6.88 & 0.3257 & 0.5707 \\
\hline & & 1.0000 & $2: 00$ & 9.4 & 9.10 & 3.18 & 0.0893 & 0.2988 \\
\hline & & 0.0413 & 4:00 & 9.2 & 9.20 & 0.00 & 0.0000 & 0.0000 \\
\hline & & 1.0000 & $6: 00$ & 8.1 & 8.14 & 0.52 & 0.0018 & 0.0424 \\
\hline & & 0.1189 & 8:00 & 8.3 & 8.30 & 0.00 & 0.0000 & 0.0000 \\
\hline & & 0.1095 & $10: 00$ & 7.1 & 7.64 & 7.65 & 0.2950 & 0.5431 \\
\hline & & 0.2280 & $12: 00$ & 8.8 & 8.80 & 0.00 & 0.0000 & 0.0000 \\
\hline & & 0.0553 & $14: 00$ & 10.3 & 10.30 & 0.00 & 0.0000 & 0.0004 \\
\hline & & 0.0914 & $16: 00$ & 10.1 & 10.56 & 4.57 & 0.2130 & 0.4615 \\
\hline & & 0.0000 & 18:00 & 12.2 & 11.48 & 5.92 & 0.5221 & 0.7226 \\
\hline & \multirow{14}{*}{ EEMD-GA-SAC } & 0.9465 & $20: 00$ & 12.2 & 11.98 & 1.77 & 0.0467 & 0.2162 \\
\hline & & 0.2387 & 22:00 & 11 & 11.00 & 0.00 & 0.0000 & 0.0002 \\
\hline & & 0.9994 & $0: 00$ & 8.3 & 8.70 & 4.79 & 0.1578 & 0.3973 \\
\hline & & 0.9927 & $2: 00$ & 9.4 & 9.26 & 1.51 & 0.0201 & 0.1419 \\
\hline & & 0.1626 & 4:00 & 9.2 & 9.20 & 0.00 & 0.0000 & 0.0000 \\
\hline & & 0.9876 & $6: 00$ & 8.1 & 8.14 & 0.52 & 0.0018 & 0.0424 \\
\hline & & 0.0290 & $8: 00$ & 8.3 & 8.30 & 0.00 & 0.0000 & 0.0000 \\
\hline & & 0.1180 & $10: 00$ & 7.1 & 7.64 & 7.56 & 0.2882 & 0.5368 \\
\hline & & 0.2376 & $12: 00$ & 8.8 & 8.80 & 0.00 & 0.0000 & 0.0000 \\
\hline & & 0.0523 & $14: 00$ & 10.3 & 10.30 & 0.00 & 0.0000 & 0.0002 \\
\hline & & 0.0928 & $16: 00$ & 10.1 & 10.54 & 4.33 & 0.1910 & 0.4370 \\
\hline & & 0.9992 & 18:00 & 12.2 & 11.58 & 5.06 & 0.3809 & 0.6171 \\
\hline & & 0.9465 & $20: 00$ & 12.2 & 11.98 & 1.77 & 0.0467 & 0.2162 \\
\hline & & 0.0536 & $22: 00$ & 11 & 11.00 & 0.01 & 0.0000 & 0.0008 \\
\hline
\end{tabular}

$$
\begin{gathered}
\widehat{b}_{t}=\frac{\alpha_{t}}{1-\alpha_{t}}\left[S_{t}^{(1)}-S_{t}^{(2)}\right], \\
\widehat{x}_{t+1}=\widehat{a}_{t}+\widehat{b}_{t}
\end{gathered}
$$

when $t=1,2, \ldots, T$, looping through Steps 2 and 3, we can get the forecasting values $\widehat{x}_{1}, \widehat{x}_{2}, \ldots, \widehat{x}_{T+1}$; when $t=T, T+$ $1, \ldots$, the forecasting values can be got by $\widehat{x}_{T+l}=\widehat{a}_{T}+\widehat{b}_{T} l$, $l=1,2, \ldots$.

Notes. If $\alpha_{1}=0$, then $\widehat{b}_{1}=0$; if $\alpha_{1}=1$, according to (11), $\widehat{b}_{1}$ is meaningless; thus let $\widehat{b}_{1}=S_{1}^{(2)}-S_{0}^{(2)}=0$. In the latter calculation, if $\alpha_{1}=1$, then $\widehat{b}_{t}=S_{t}^{(2)}-S_{t-1}^{(2)}$. In addition, based on (10) it is found that if $\alpha_{t}<1$ at a certain time, then $\alpha_{t}<1$ at any time in the future, and $\widehat{b}_{t}$ can be calculated by (11).

3.5. Hybrid EEMD-GA-FAC/SAC Model. As exponential smoothing methods, first-order adaptive coefficient forecasting method and second-order adaptive coefficient forecasting method have been commonly used for short-term and medium-term time-series trend forecast. The exponential smoothing is compatible with the advantage of entire period average and the moving average, which utilize the history data to affect the weight gradually. It converges to zero as far away from the data. It is set equal to 0.2 or 0.1 in terms of experience that the smoothing parameter of the first-order adaptive coefficient forecasting method and second-order adaptive coefficient forecasting method under normal circumstances. However, neither of them is a universal model that is appropriate for all circumstances; therefore the parameter should be adjusted to different situations. Moreover, if the original data was preprocessed with high frequency interference information eliminated before employing exponential smoothing method, it would be more attributed to make prediction. As the topic of this paper, wind speed forecasting problem shows nonstationarity with high frequency interference information and can be settled by exponential smoothing method of which the smoothing parameter gets optimized. In view of the above two points, it is necessary to develop a hybrid methodology to make full use of the advantages of respective methods. The combining methodology consisted of four steps.

Step 1. EEMD method is used to preprocess the original data before employing model.

Step 2. FAC and SAC are employed to do forecast using the preprocessed data by EEMD method. 
Step 3. Genetic algorithm is introduced to determine the optimal weight parameter instead of experiential value.

Step 4. Evaluate the forecasting performance and effects of the models.

\section{Experimental Simulation and Results Analysis}

4.1. Data Description. Shandong Peninsula (shown in Figure 2) is located in the northeast of Shandong Province in China. As an economy-developed and large energy consumption province, Shandong Province wind power industry can greatly mitigate the high pressure of energy conservation and emissions reduction. Therefore, wind energy evaluation and estimation in Shandong Province are difficult but quite important task, which contribute to the grid interconnection of wind farms, saving energy consumption, and reducing pollution.

The forecasting methods, forecasting horizons, and the certain locations of wind speed properties all have impact on wind speed forecasts. As a whole, the shorter forecasting horizons generally ease the change of wind speed, thus getting smaller forecasting errors than middle- or long-term forecast [33]. In this paper, the ten-minute wind speed is selected in Yantai, Weihai, and Qingdao of Shandong Peninsula. The locations of the three selected observation stations are shown in Figure 2. The collected data is from June 1, 2011, to June 6,2011 , between 00:00 and 23:50, with ten-minute interval each day for model construction and model test. Figure 3 shows the variation trend of wind speed. It can be seen that wind speed has large random fluctuation characteristics with nonlinear and nonstationary signals. Therefore, it is essential to employ EEMD method to eliminate the high frequency information of ten-minute wind speed before constructing the model, which not only reveals the inner nature of the data but also improves forecasting accuracy.

4.2. Data Processing. In financial econometrics, the noise signal is dominant in high frequency data, so the authors prefer low frequency data rather than fine sampled data to obtain more stable estimates [35]. If the data with nonstationary signals and high frequency inference information is used to establish the forecasting model, it will cause worse forecasting results. Thus, ensemble empirical mode decomposition (EEMD) is employed to eliminate the high frequency interference information in original ten-minute wind speed series. According to the algorithm of EEMD method, it decomposes the collected wind speed with noiseadded signal into nine IMFs, removes the first IMF, and calculates the sum of the rest of the IMFs. Through data processing, it can be seen that the curve of the denoising data is smoother than the original wind speed time series in Figure 4 (taking the first training set from 1 June 2011 at 0:00 to 6 June 2011 at 23:50 as an example).

Furthermore, the processed data are applied to establish model and assess the forecasting quality and effects of the models.

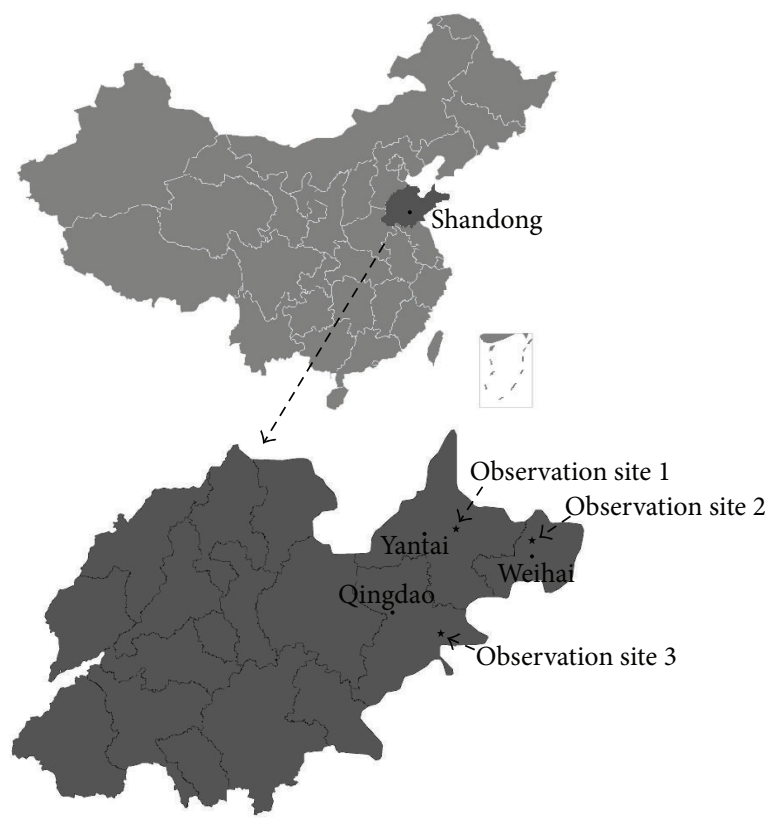

\begin{tabular}{lcc}
\hline Area & East longitude & North latitude \\
\hline Observation site 1 & $121^{\circ} 39^{\prime}$ & $37^{\circ} 52^{\prime}$ \\
Observation site 2 & $122^{\circ} 10^{\prime}$ & $37^{\circ} 50^{\prime}$ \\
Observation site 3 & $120^{\circ} 33^{\prime}$ & $36^{\circ} 07^{\prime}$ \\
\hline
\end{tabular}

FIGURE 2: The geographical location of the three observation sites in Shandong Peninsula.
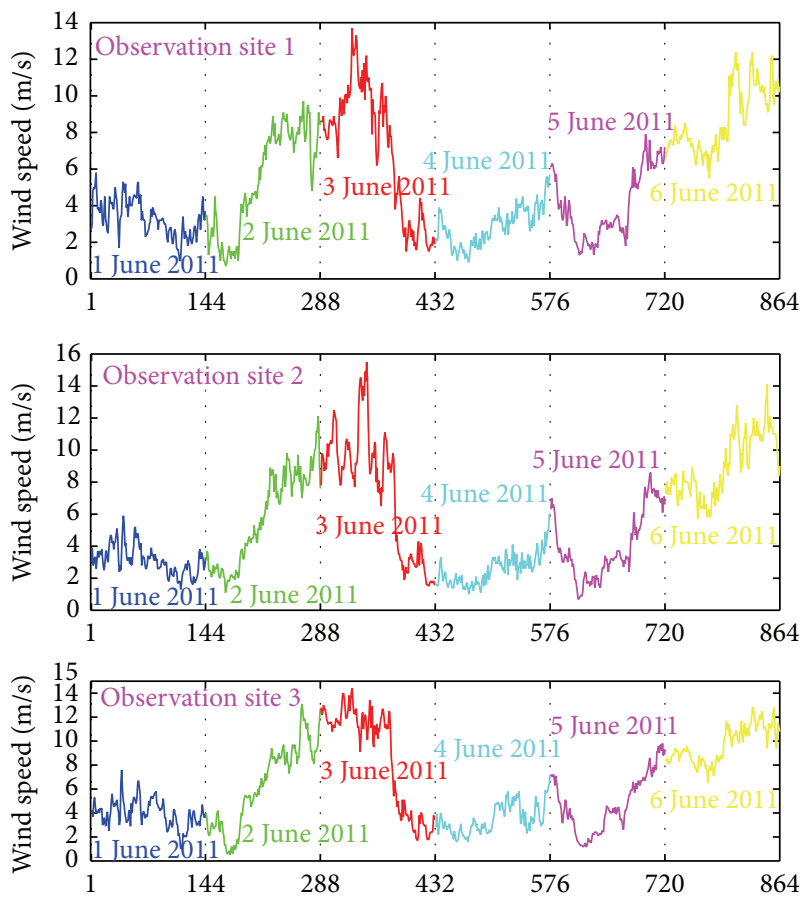

FIGURE 3: Original wind speed data collected every $10 \mathrm{~min}$ from June 1,2011 , to June 6,2011 , in the three observations sites. 

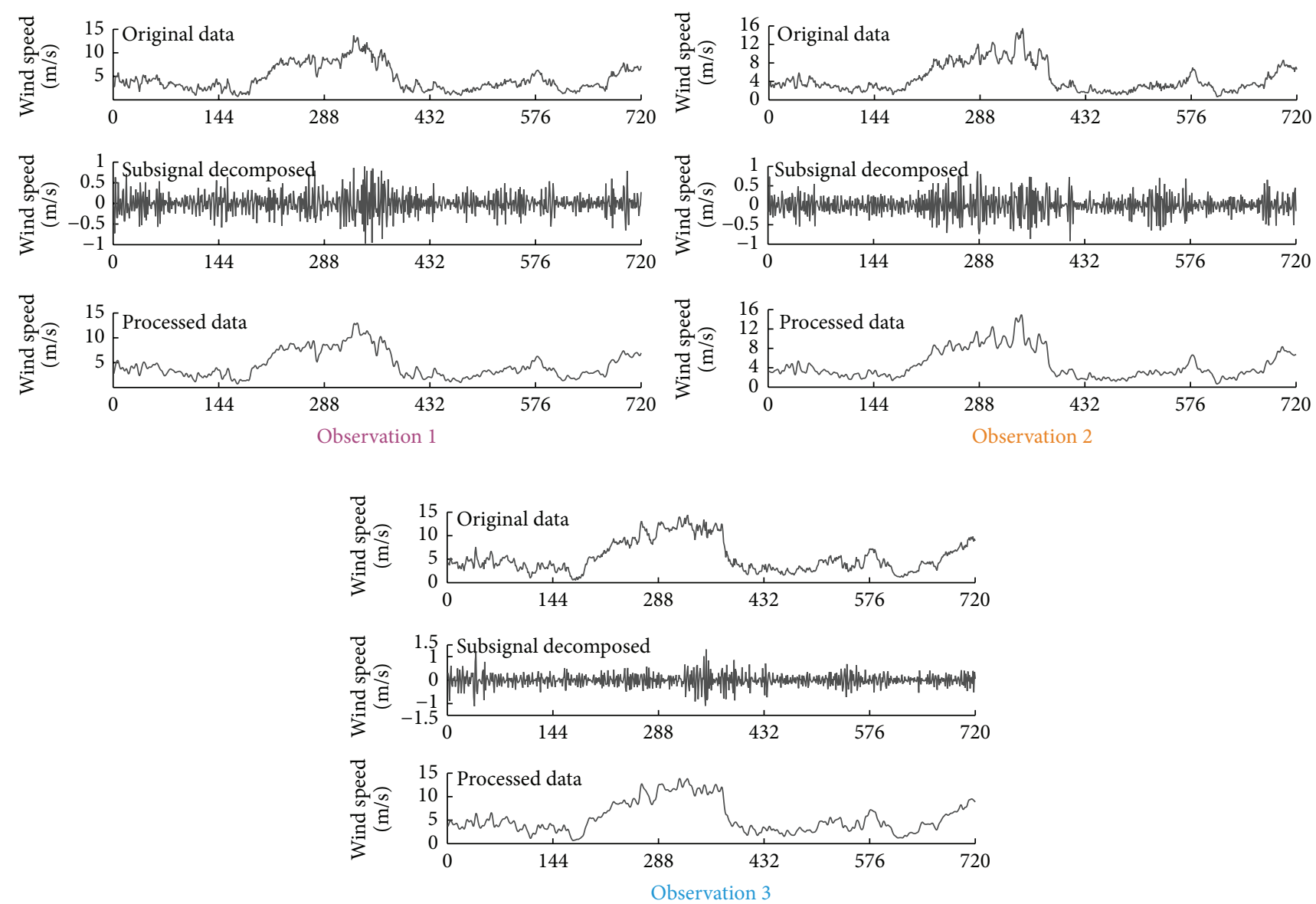

FIGURE 4: Denoising process for the original wind speed data in the three observation sites.

4.3. Statistical Measurements of Forecasting Quality. Yokum and Armstrong concluded that the accuracy criterion was more important in comparison with cost savings generated from improved methods and execution issues; they conducted an expert opinion survey about the evaluation measurements in order to select forecasting techniques [36].

To evaluate the forecasting quality and effects of the models quantitatively, we utilize multiple statistical measurements including the mean absolute percentage error (MAPE), mean square error (MSE), and mean absolute error (MAE). When these three forecasting errors decrease, the accuracies of the forecasting results will increase $[37,38]$. They are calculated as follows:

$$
\begin{gathered}
\text { MAPE }=\frac{1}{n} \sum_{t=1}^{n}\left|e_{t}\right|=\frac{1}{n} \sum_{t=1}^{n} \frac{\left|x_{t}-\widehat{x}_{t}\right|}{x_{t}} \times 100 \%, \\
\operatorname{MSE}=\frac{1}{n} e_{t}^{2}=\frac{1}{n} \sum_{t=1}^{n}\left(x_{t}-\widehat{x}_{t}\right)^{2}, \\
\operatorname{MAE}=\frac{1}{n}\left|e_{t}\right|=\frac{1}{n} \sum_{t=1}^{n}\left|x_{t}-\widehat{x}_{t}\right|,
\end{gathered}
$$

where $e_{t}=x_{t}-\hat{x}_{t}, t$ corresponds to the sample size, $x_{t}$ represents the actual value at time $t$, and $\widehat{x}_{t}$ represents the forecasting value at time $t$.
4.4. Results and Discussion of Traditional Models. Due to the large random fluctuations of wind speed, wind power grid is a difficult and challenging task. It can effectively calculate the spinning reserve capacity of grid security forewarning management in wind grid that accurately forecasts the wind speed every two hours, which can ensure the safe operation of the grid. In order to make the wind speed forecasts at integral point moment with a certain time interval of 2 hours on 6 June 2011 from 00:00 to 22:00, the original wind speed data of each observation site are grouped into twelve training sets. The data in the first training set is from 0:00 on 1 June to 23:50 on 5 June, the second training set is from 2:00 on 1 June to $1: 50$ on 6 June, and the third training set is from 4:00 on 1 June to 3:50 on 6 June. Correspondingly, according to the same grouping principle to form the remaining training set, the twelfth training set is from 22:00 on 1 June to 21:50 on 6 June with 720 pieces of data in each training set. The test data set is formed by the integral point time moment on 6 June in 2011, which begins at 0:00 with the certain interval of two hours. It is used to test the effectiveness of the optimized hybrid models.

In this section, the forecasting models including FAC, SAC, BP, and ARIMA models can be compared. Tables 1 and 2 show the forecasting results and the forecasting accuracies of four different traditional models. As displayed in Table 2, 
in observation site 1 , the MAPEs of BP and ARIMA are above $6 \%$ and the MAPEs of FAC and SAC are below $6 \%$. The MAEs of FAC and SAC are considerably lower than the ones of BP and ARIMA model. Therefore, it is presented that FAC and SAC model perform better than BP and ARIMA model in MAPE and MAE. For observation site 3, comparing with the MAPE, MSE, and MAE of BP and ARIMA, FAC and SAC still have higher forecasting accuracies. However, for observation site 2, it is not difficult to find that BP and ARIMA show better forecasting performance. Consequently, the forecasting abilities of four traditional models exhibit different results for different sites. In addition, it can also be found that the forecasting qualities of FAC and SAC model are similar.

4.5. Results and Discussion of Different Hybrid Models. In this section, the hybrid models including EEMD-FAC/SAC and EEMD-GA-FAC/SAC are compared. The original wind speed data can be preprocessed by EEMD method, which aims to eliminate the high frequency nonstationary information. After data preprocessing, the amount of twelve training sets is grouped in the same manner presented in Section 4.4. Correspondingly, the forecasting results of EEMD-FAC/SAC are described in Table 3. It is from 00:00 to 22:00 on 6 June with a certain interval of two hours. Then genetic algorithm is utilized to determine the optimal weight parameter to predict.

The forecasting results of the proposed hybrid EEMDGA-FAC/SAC model based on the processed data and the values of weight parameter $\beta$ from three observation sites are shown in Tables 4-6. The detailed procedures of the hybrid EEMD-FAC/SAC model and the proposed hybrid model EEMD-GA-FAC/SAC are presented by the following.

Step 1. Decompose the nonstationary high frequency information from the original wind speed series.

Step 2. Produce the forecast results of FAC and SAC models utilizing the processed data by EEMD method, which is represented by EEMD-FAC/SAC.

Step 3. Optimize the forecasting results of hybrid EEMDFAC/SAC model by selecting the best parameter $\beta$ with genetic algorithm.

Step 4. Analyze the forecasting performance among the hybrid EEMD-FAC/SAC model and the proposed hybrid EEMD-GA-FAC/SAC model.

It is easy to find that the results of three forecasting errors obtained by the FAC/SAC model are higher than those of EEMD-FAC/SAC model and EEMD-GA-FAC/SAC model in all the observation sites. In addition, it is also clear to find that the statistical forecasting errors produced by EEMD-FAC/SAC model are higher than those of EEMDGA-FAC/SAC model. Hence, the proposed hybrid EEMDGA-FAC/SAC model presents better forecasting accuracy than the hybrid EEMD-FAC/SAC model and the traditional FAC/SAC model. It is illustrated that the proposed hybrid
TABLE 7: Errors comparisons of all forecasting models in site 1.

\begin{tabular}{lccc}
\hline Models & MAPE $(\%)$ & MSE $\left(\mathrm{m}^{2} / \mathrm{s}^{2}\right)$ & MAE $(\mathrm{m} / \mathrm{s})$ \\
\hline BP & 6.0643 & 0.5808 & 0.5432 \\
ARIMA & 6.1497 & 0.5814 & 0.5588 \\
FAC & 4.6603 & 0.6668 & 0.4562 \\
EEMD-FAC & 4.3615 & 0.6288 & 0.4301 \\
EEMD-GA-FAC & 3.1165 & 0.3357 & 0.3050 \\
SAC & 5.3317 & 0.7595 & 0.5356 \\
EEMD-SAC & 4.3776 & 0.6350 & 0.4319 \\
EEMD-GA-SAC & 2.6109 & 0.3027 & 0.2622 \\
\hline
\end{tabular}

TABLE 8: Errors comparisons of all forecasting models in site 2.

\begin{tabular}{lccc}
\hline Models & MAPE $(\%)$ & MSE $\left(\mathrm{m}^{2} / \mathrm{s}^{2}\right)$ & MAE $(\mathrm{m} / \mathrm{s})$ \\
\hline BP & 5.8862 & 0.4006 & 0.5245 \\
ARIMA & 5.8202 & 0.4135 & 0.5280 \\
FAC & 6.2611 & 0.4150 & 0.5758 \\
EEMD-FAC & 5.9313 & 0.3865 & 0.5398 \\
EEMD-GA-FAC & 2.7217 & 0.1063 & 0.2476 \\
SAC & 6.4436 & 0.4339 & 0.5927 \\
EEMD-SAC & 6.0198 & 0.3893 & 0.5487 \\
EEMD-GA-SAC & 5.1493 & 0.3526 & 0.4448 \\
\hline
\end{tabular}

model can take advantage of the characteristics of the single model and is less sensitive.

4.6. Comprehensive Comparison of the Hybrid Model and Traditional Models. The detailed procedure of the proposed hybrid model EEMD-GA-FAC/SAC for wind speed forecast can be displayed in Figure 5. The errors comparisons of all forecasting models can be seen in Tables 7-9. Taking site 1 as an example to analyze, the proposed hybrid EEMDGA-FAC model results in $2.9478 \%, 3.0332 \%, 1.5438 \%$, and $1.2450 \%$ reductions in MAPE, $0.2451,0.2457,0.3311$, and 0.2931 reductions in MSE, and $0.2382,0.2538,0.1512$, and 0.1251 reductions in MAE compared to BP, ARIMA, FAC, and EEMD-FAC. The proposed hybrid EEMD-GA-SAC model leads to $3.4534 \%, 3.5388 \%, 2.7208 \%$, and $1.7667 \%$ reductions in MAPE, $0.2781,0.2787,0.4568$, and 0.3323 reductions in MSE, and $0.2810,0.2966,0.2734$, and 0.1697 reductions in MAE compared to BP, ARIMA, SAC, and EEMD-SAC. For sites 2 and 3, the proposed hybrid model EEMD-GAFAC/SAC also presents the best forecasting effects in all the models.

Through further analysis, it is found that EEMD-GA-FAC model is better than EEMD-GA-SAC model in observation site 2, but EEMD-GA-FAC model is worse than EEMDGA-FAC model in observation sites 1 and 3. It is illustrated that, for different types of data, EEMD-GA-FAC model and EEMD-GA-SAC model present different forecasting quality. However, no matter which is the best forecasting model, it is concluded that our proposed hybrid model EEMD-GAFAC/SAC outperforms other traditional models and hybrid 


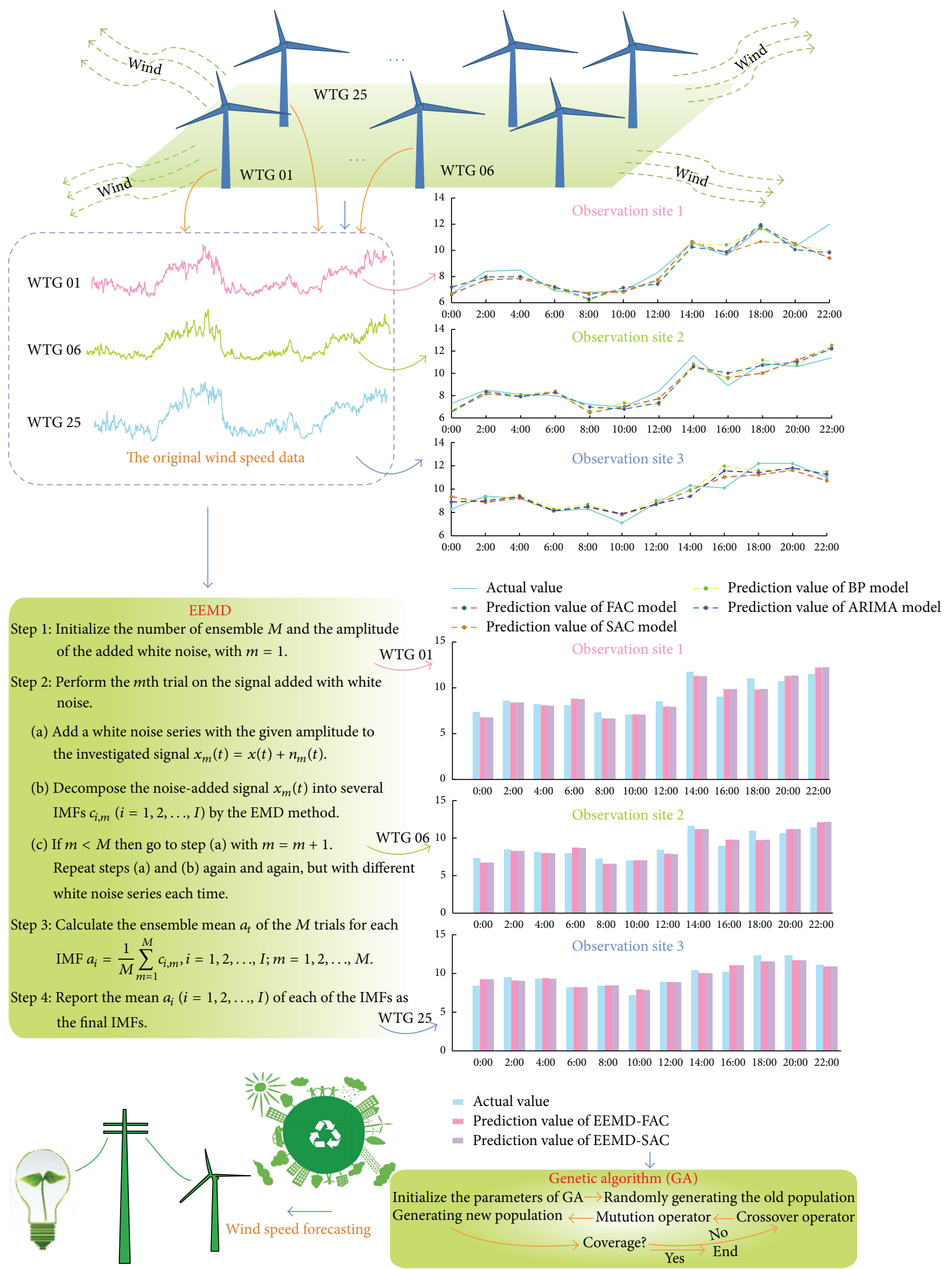

FIGURE 5: Flowchart of the proposed hybrid model EEMD-GA-FAC/SAC. 
TABLE 9: Errors comparisons of all forecasting models in site 3.

\begin{tabular}{lccc}
\hline Models & MAPE $(\%)$ & MSE $\left(\mathrm{m}^{2} / \mathrm{s}^{2}\right)$ & $\mathrm{MAE}(\mathrm{m} / \mathrm{s})$ \\
\hline BP & 5.7011 & 0.4848 & 0.5421 \\
ARIMA & 5.2878 & 0.4184 & 0.5066 \\
FAC & 5.0800 & 0.3593 & 0.4847 \\
EEMD-FAC & 4.3924 & 0.2793 & 0.4153 \\
EEMD-GA-FAC & 2.5415 & 0.1245 & 0.2380 \\
SAC & 5.0617 & 0.3610 & 0.4840 \\
EEMD-SAC & 4.3865 & 0.2796 & 0.4159 \\
EEMD-GA-SAC & 2.1289 & 0.0905 & 0.1991 \\
\hline
\end{tabular}

models. To sum up, the proposed hybrid model EEMD-GAFAC/SAC is suitable to forecast wind speed with a certain time interval of 2 hours in Shandong Peninsula of China.

\section{Conclusions}

Wind speed forecasting becomes increasingly important for wind farm management and the conversion of wind power in power dispatch of hybrid system. Therefore, this paper proposes an intelligent optimized hybrid model based on EEMD, FAC or SAC, and GA to forecast wind speed in Shandong Peninsula of China. This hybrid model uses EEMD method to decompose the noise and eliminate the high frequency interference information of the original wind speed data and applies an artificial intelligent optimization algorithm GA to determine the optimal parameter of FAC and SAC model. As a case study, every ten-minute wind speed data from 1 June to 6 June in 2011 in three observation sites of Shandong Peninsula are collected and multiple errors evaluation criteria like MAPE, MSE, and MAE are chosen to validate the forecasting performance of the hybrid model. The experimental results show that the hybrid model has the best forecasting performance in comparison with traditional models like BP, ARIMA, FAC, SAC, EEMD-FAC, and EEMD-SAC, from which it can be concluded that the proposed hybrid model EEMD-GA-SAC/FAC can effectively, adaptively, and reliably improve the forecasting performance in large wind farms of China.

\section{Conflict of Interests}

The authors declare that there is no conflict of interests regarding the publication of this paper.

\section{Acknowledgments}

This work is supported by the Government Special Funds for Local Colleges and Universities Development under Grants DUFE2014J29 and DUFE2014Q52. The authors appreciate the comments and suggestions provided by Jianzhou Wang and Jinrui Wei.

\section{References}

[1] Z. H. Guo, Y. Dong, J. Z. Wang, and H. Y. Lu, "The forecasting procedure for long-term wind speed in the Zhangye area," Mathematical Problems in Engineering, vol. 2010, Article ID 684742, 17 pages, 2010.

[2] G. Xydis, C. Koroneos, and M. Loizidou, "Exergy analysis in a wind speed prognostic model as a wind farm sitting selection tool: a case study in Southern Greece," Applied Energy, vol. 86, no. 11, pp. 2411-2420, 2009.

[3] A. Ucar and F. Balo, "Investigation of wind characteristics and assessment of wind-generation potentiality in Uludağ-Bursa, Turkey," Applied Energy, vol. 86, no. 3, pp. 333-339, 2009.

[4] M. Monfared, H. Rastegar, and H. M. Kojabadi, "A new strategy for wind speed forecasting using artificial intelligent methods," Renewable Energy, vol. 34, no. 3, pp. 845-848, 2009.

[5] P. Pinson, H. A. Nielsen, H. Madsen, and G. Kariniotakis, "Skill forecasting from ensemble predictions of wind power," Applied Energy, vol. 86, no. 7-8, pp. 1326-1334, 2009.

[6] G. Li and J. Shi, "On comparing three artificial neural networks for wind speed forecasting," Applied Energy, vol. 87, no. 7, pp. 2313-2320, 2010.

[7] R. G. Kavasseri and K. Seetharaman, "Day-ahead wind speed forecasting using f-ARIMA models," Renewable Energy, vol. 34, no. 5, pp. 1388-1393, 2009.

[8] H. P. Liu, E. Erdem, and J. Shi, "Comprehensive evaluation of ARMA-GARCH(-M) approaches for modeling the mean and volatility of wind speed," Applied Energy, vol. 88, no. 3, pp. 724732,2011

[9] C. González-Mingueza and F. Muñoz-Gutiérrez, "Wind prediction using Weather Research Forecasting model (WRF): a case study in Peru," Energy Conversion and Management, vol. 81, pp. 363-373, 2014.

[10] S. Janjai, I. Masiri, W. Promsen et al., "Evaluation of wind energy potential over Thailand by using an atmospheric mesoscale model and a GIS approach," Journal of Wind Engineering and Industrial Aerodynamics, vol. 129, pp. 1-10, 2014.

[11] L. Ma, S. Y. Luan, C. W. Jiang, H. L. Liu, and Y. Zhang, "A review on the forecasting of wind speed and generated power," Renewable and Sustainable Energy Reviews, vol. 13, no. 4, pp. 915-920, 2009.

[12] Z. Guo, W. Zhao, H. Lu, and J. Wang, "Multi-step forecasting for wind speed using a modified EMD-based artificial neural network model," Renewable Energy, vol. 37, no. 1, pp. 241-249, 2012.

[13] J. Wang, W. Zhu, W. Zhang, and D. Sun, "A trend fixed on firstly and seasonal adjustment model combined with the $\varepsilon$-SVR for short-term forecasting of electricity demand," Energy Policy, vol. 37, no. 11, pp. 4901-4909, 2009.

[14] S. A. Pourmousavi Kani and M. M. Ardehali, "Very short-term wind speed prediction: a new artificial neural network-Markov chain model," Energy Conversion and Management, vol. 52, no. 1, pp. 738-745, 2011.

[15] Z. Guo, J. Zhao, W. Zhang, and J. Wang, "A corrected hybrid approach for wind speed prediction in Hexi Corridor of China," Energy, vol. 36, no. 3, pp. 1668-1679, 2011.

[16] J. Wang, S. Zhu, W. Zhang, and H. Lu, "Combined modeling for electric load forecasting with adaptive particle swarm optimization," Energy, vol. 35, no. 4, pp. 1671-1678, 2010.

[17] H. Hariharan, A. Gribok, M. A. Abidi, and A. Koschan, "Image fusion and enhancement via empirical mode decomposition," 
Journal of Pattern Recognition Research, vol. 1, no. 1, pp. 16-31, 2006.

[18] R. Ditommaso, M. Mucciarelli, S. Parolai, and M. Picozzi, "Monitoring the structural dynamic response of a masonry tower: comparing classical and time-frequency analyses," Bulletin of Earthquake Engineering, vol. 10, no. 4, pp. 1221-1235, 2012.

[19] Y. Dong, J. Wang, H. Jiang, and J. Wu, "Short-term electricity price forecast based on the improved hybrid model," Energy Conversion and Management, vol. 52, no. 8-9, pp. 2987-2995, 2011.

[20] N. S. Kim, K. Chung, S. Ahn, J. W. Yu, and K. Choi, “Denoising traffic collision data using Ensemble Empirical Mode Decomposition (EEMD) and its application for constructing Continuous Risk Profile (CRP)," Accident Analysis \& Prevention, vol. 71, pp. 29-37, 2014.

[21] E. Erdem and J. Shi, "ARMA based approaches for forecasting the tuple of wind speed and direction," Applied Energy, vol. 88, no. 4, pp. 1405-1414, 2011.

[22] J. Yuan, C. He, W. Gao, J. Lin, and Y. Pang, "A novel hard decision decoding scheme based on genetic algorithm and neural network," Optik, vol. 125, no. 14, pp. 3457-3461, 2014.

[23] B. M. Battista, A. D. Addison, and C. C. Knapp, "Empirical mode decomposition operator for dewowing GPR data," Journal of Environmental and Engineering Geophysics, vol. 14, no. 4, pp. 163-169, 2009.

[24] H. C. Wang, J. Chen, and G. M. Dong, "Feature extraction of rolling bearing's early weak fault based on EEMD and tunable Q-factor wavelet transform," Mechanical Systems and Signal Processing, vol. 48, no. 1-2, pp. 103-119, 2014.

[25] T. Wang, M. Zhang, Q. Yu, and H. Zhang, "Comparing the applications of EMD and EEMD on time-frequency analysis of seismic signal," Journal of Applied Geophysics, vol. 83, pp. 29-34, 2012.

[26] Z. H. Wu and N. E. Huang, "Ensemble empirical mode decomposition: a noise-assisted data analysis method," Tech. Rep., Center for Ocean-Land-Atmosphere Studies, 2005.

[27] Z. Wu and N. E. Huang, "Ensemble empirical mode decomposition: a noise-assisted data analysis method," Advances in Adaptive Data Analysis, vol. 1, no. 1, pp. 1-41, 2009.

[28] A. Sokolov and D. Whitley, "Unbiased tournament selection," in Proceedings of the Genetic and Evolutionary Computation Conference (GECCO '05), pp. 1131-1138, ACM, Washington, DC, USA, June 2005.

[29] S. M. Homayouni, S. H. Tang, and O. Motlagh, "A genetic algorithm for optimization of integrated scheduling of cranes, vehicles, and storage platforms at automated container terminals," Journal of Computational and Applied Mathematics, vol. 270, pp. 545-556, 2014.

[30] Varun and Siddhartha, "Thermal performance optimization of a flat plate solar air heater using genetic algorithm," Applied Energy, vol. 87, no. 5, pp. 1793-1799, 2010.

[31] A. Azadeh and S. Tarverdian, "Integration of genetic algorithm, computer simulation and design of experiments for forecasting electrical energy consumption," Energy Policy, vol. 35, no. 10, pp. 5229-5241, 2007.

[32] W. Zhang, J. Wu, J. Wang, W. Zhao, and L. Shen, "Performance analysis of four modified approaches for wind speed forecasting," Applied Energy, vol. 99, pp. 324-333, 2012.

[33] X. Yang, Y. Xiao, and S. Chen, "Research on wind speed and power generation forecasting in wind farm," in Proceedings of the Chinese Society for Electrical Engineering (CSEE '05), vol. 25, pp. 1-5, 2005, (Chinese).

[34] D. X. Niu, S. H. Cao, L. Zhao, and W. W. Zhang, Power Load Forecasting Technique and Its Application, China Electric Power Press, Beijing, China, 1998.

[35] L. Zhang, P. A. Mykland, and Y. Aï-Sahalia, "A tale of two time scales," Journal of the American Statistical Association, vol. 100, no. 472, pp. 1394-1411, 2005.

[36] J. T. Yokuma and J. S. Armstrong, "Beyond accuracy: comparison of criteria used to select forecasting methods," International Journal of Forecasting, vol. 11, no. 4, pp. 591-597, 1995.

[37] W. G. Zhao, J. Z. Wang, and H. Y. Lu, "Combining forecasts of electricity consumption in China with time-varying weights updated by a high-order Markov chain model," Omega, vol. 45, pp. 80-91, 2014.

[38] J. Z. Wang, Y. Dong, and H. Jiang, "A study on the characteristics, predictions and policies of China's eight main power grids," Energy Conversion and Management, vol. 86, pp. 818-830, 2014. 


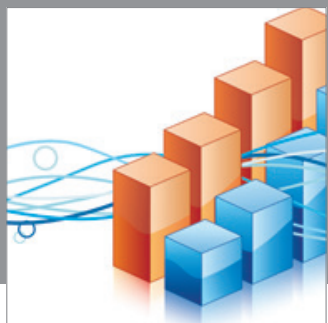

Advances in

Operations Research

mansans

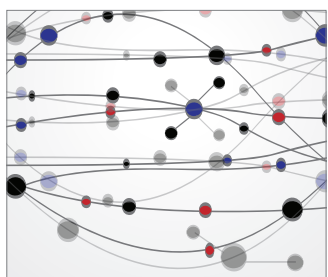

The Scientific World Journal
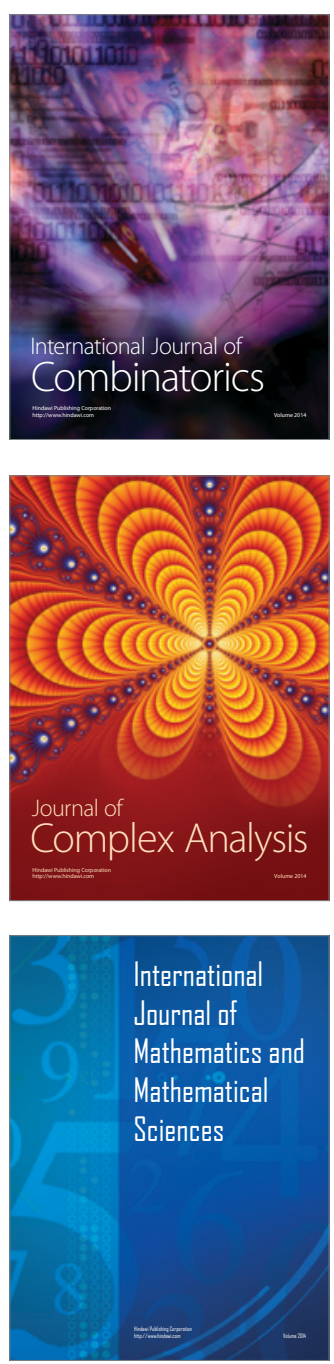
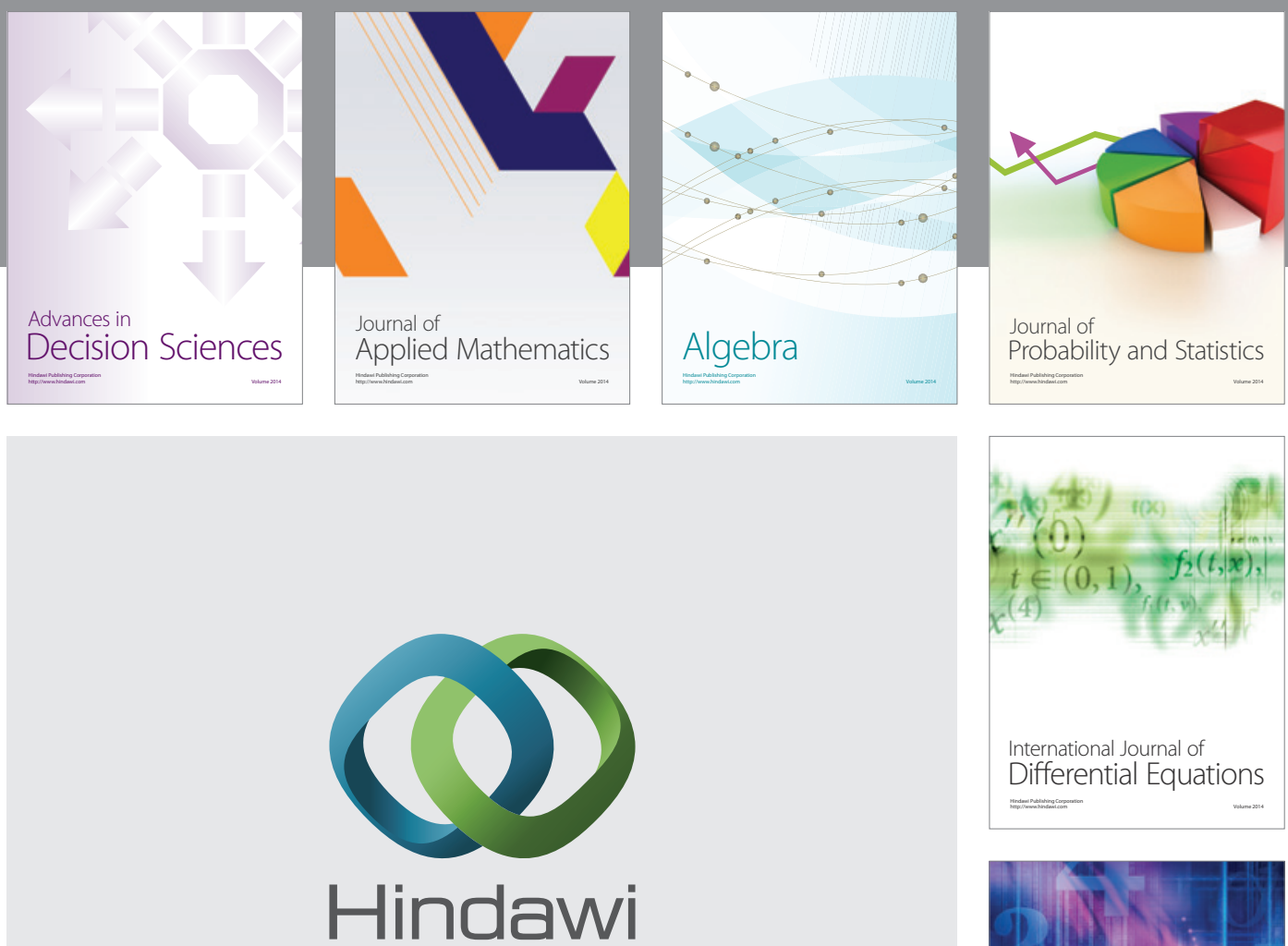

Submit your manuscripts at http://www.hindawi.com
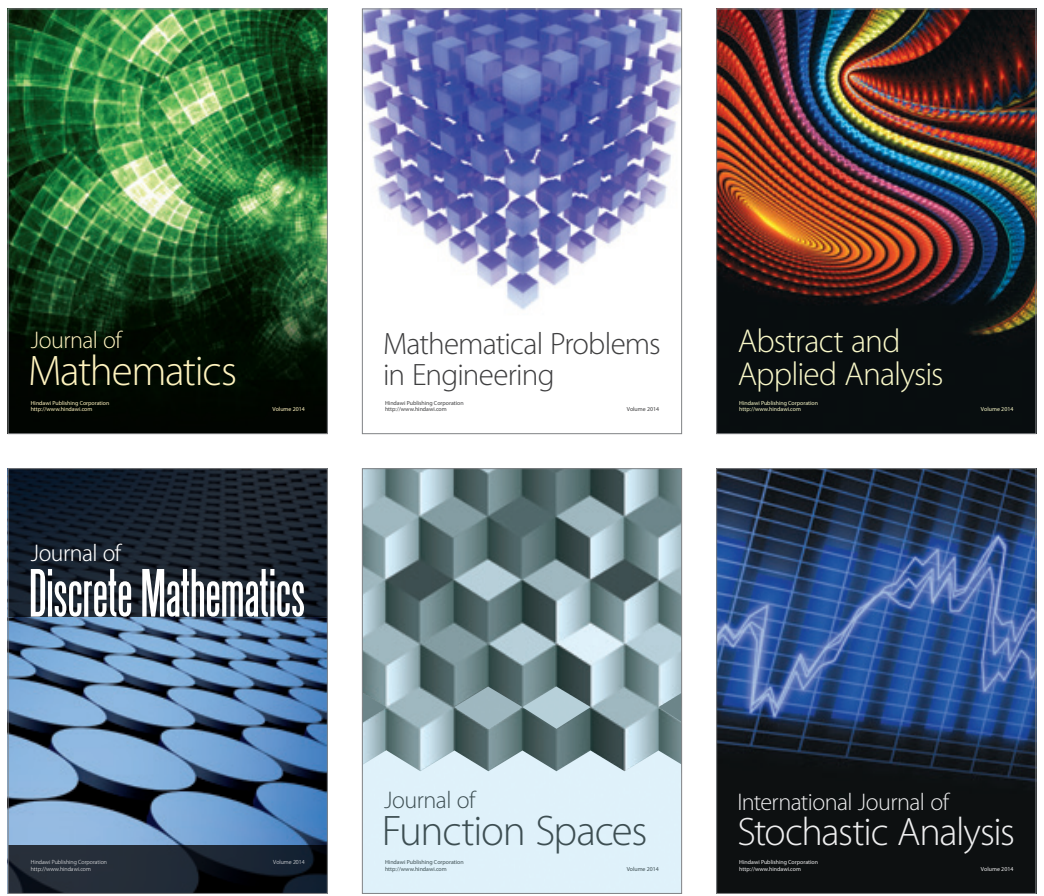

Journal of

Function Spaces

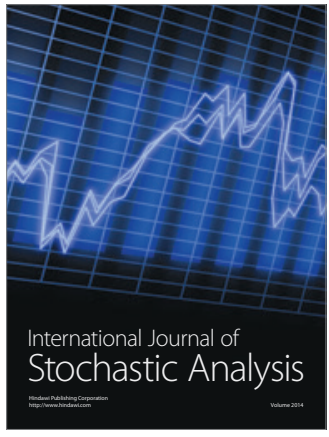

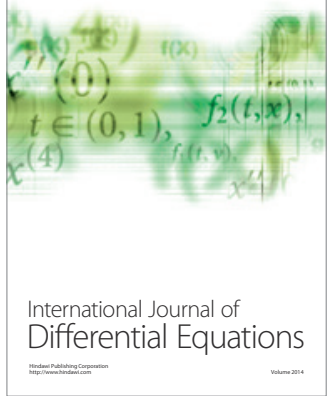
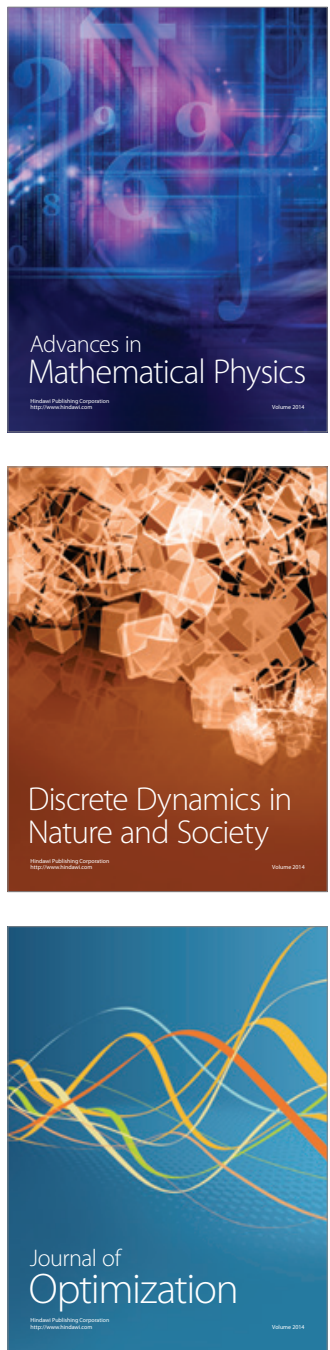